JASC 12-2-10

\title{
Clinical Information Interchange System using HL7-CDA
}

\author{
Yong Gyu Jung ${ }^{\dagger}$, Young Ho Lee* \\ 'Dept. of Medical IT Marketing, Eulji University, Korea \\ * Dept. of Computer Science Suwon University, Korea
}

\begin{abstract}
In highly developed society, information and communication technologies are widely used for better medical services. These information and communication technologies should be more and more acceptable in all hospitals for exchange medical records. EMR becomes more convenient than the previously used paper charts. It will be able to record medical institutions every time and dual treatment. Each is different specifications for each medical institution to use the program or document to exchange it. The personal clinic records still does not exchange well. To solve this gap between medical alienation, this paper describes the concepts of HL7-CDA and proposes types of telemedicine system. To resolve time and space constraints, new form of treatment methods presents in future directions after described about related systems. CDA enables electronic medical records to the each medical center and gradually expanded by exchanging the patient's medical records. This paper is using XML-based CDA documents as a hierarchical for medical information exchange standards compliant HL7-CDA documents. It could be possible currently used structural variety of multimedia data. Thus It is able to send and receive HL7-CDA-based medical information and clinical information to identify the medical institutions of medical information with interchange system design and building standards, and through mutual exchange of clinical information.
\end{abstract}

Key words: HL7, CDA, XML, medical information, clinical information

\section{INTRODUCTION}

Standardization work is increasing for a lot of messages and documents exchange according to the computerization. It has been actively performed for the mutual exchange of information and various related agencies and hospitals. For jumping beyond the traditional way of documents, Electronic recording and exchanging system are needed for medical documents processing. HL7 was founded for the purpose of exchanging medical information between hospitals as a standardization body. It defines the various messages associated for hospital administration works and medical care. In general Hospital, the mutual exchanges of medical information including the patients' medical information are increasingly in growing numbers. HL7-CDA is a standard format in creating and staring the information on the local system, as well as in interchanging between different healthcare sectors. The software applications to be compatible with a set of rules in a form

Manuscript received : Sept. 28, 2012 / revised : Nov. 1, 2012

${ }^{\dagger}$ Corresponding Author: ygjung@eulji.ac.kr

Tel: +82-31-740-7178, Fax: +82-31-740-7178,

Dept. of Medical IT Marketing, Eulji University Korea and a formal agreement with the ISO TC215 as the consensus standard jointly developed by the users and system providers, and other medical information to officials and representatives. Industry groups to participate in the current HL7 standard are medical service providers, pharmacies, government health agencies and departments, health care sectors and software companies. XML-based CDA is currently being used in HL7 interchange of medical information in the way HL7-CDA, It is based on the existing HL7 interchange of medical information, unlike the data exchange and management of HL7-CDA-based medical system designed for information interchange.

In this paper, beyond the existing medical information in text format, image, or a variety of multimedia information data exchange and management of each agency based on the HL7-CDA for the mutual exchange of medical information and clinical information system to point out the problems and limitations accordingly. To overcome inefficient competition in information sharing between hospitals reducing the burden of patients with molting, it is an urgent need to ensure the soundness. Also to reduce duplicate investment, ensure compatibility between the hospital 
system and hospital consultations between the governments under the leadership must promote the true interests of the patient.

\section{RELATED RESEARCH}

EMR is a way to eliminate paper documents used in hospitals to computerize all the data stored on the media. Currently widely used until the paper duty record for a long time collecting the ground sometime in the hospital most of the permanent preservation of medical records management and storage limitations on the import care after a period of time, the last non-utilization of medical records in the past, microfilm, in recent years management and optical discs, compact discs, and more. Connection network linking them Computerization Division and consists of an image consisting only of an electronic medical record storage and retrieval purposes can be utilized on a limited basis in the early stages of an electronic medical record.

\subsection{HL7}

HL7 has the event-driven protocols to transfer information on message basis system corresponding to the OSI 7 layer standards with the application. Therefore, It supports different medical data acquisition and communication for promotion and application. HL7 basically must be met and followed the five security issues.

1 Certification: The first intention of communication between the parties can be a certified.

2. Confidentiality of information by unauthorized communication between the parties to prevent leaks and revelations.

3. Stewardship can be checked at any time and must be also guaranteed automatically legally binding on the results.

4. Whether to change the information when the information to be exchanged by a three-character integrity proof: non-authenticated should prove possible,

5. Data of the sender and the receiver without all contradiction proven to be capable of non-repudiation. Efficient cryptographic techniques HL7 5 things to satisfy security issues still need to maintain the same security for authentication, confidentiality.

\subsection{HL7 CDA}

With different types of document to use in patient care, standard form required to exchange documents between agencies. CDA is used for exchange documents to standardize the format. As the purpose of the standard HL7-CDA in medical information, It is derived and exchanged as electronic records with standards. HL7-CDA has the following features.
1 The clinical documentation shall maintain the document as unchanged.

2 It shall be maintained by the designated person or organization.

3 Clinical information of the document is consists of a set of authentication information legally.

4 Clinical documents could be applied in the context of the basic authentication configuration and the contents of the document.

5 Human clinical documents must be able to read.

HL7-CDA document header contains the classification of documents, verification, certification, patients, health professionals involved in information exchange of the hospital or hospital records. At the same time it has the advantages to facilitate the management. Clinical report contained a non-structural or structural markup in the body can be constructed as shown in figure 1 .

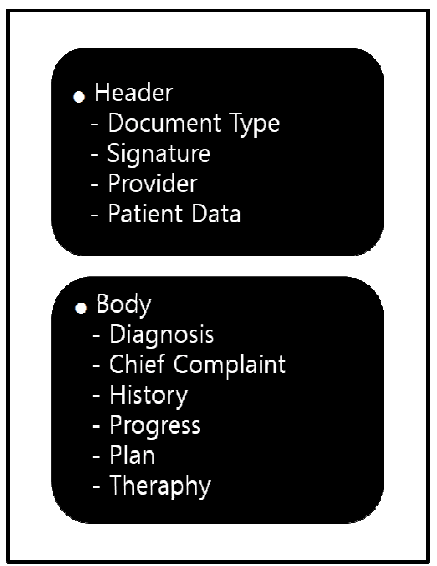

Figure 1. The structure of the HL7-CDA

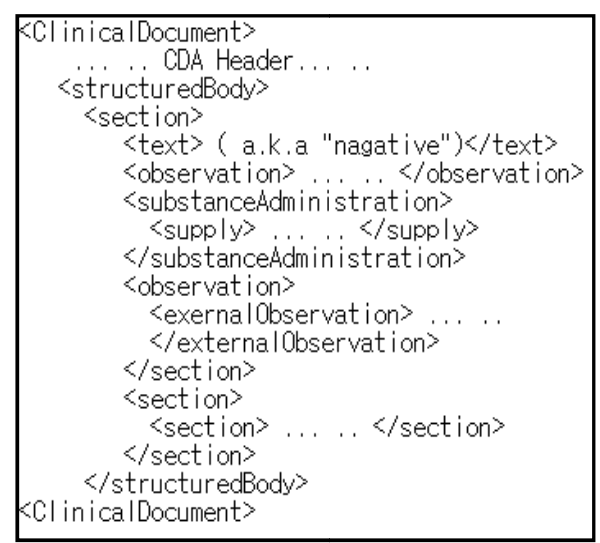

Figure 2. CDA's main components.

All patients' medical information provides an interchangeable CDA. Therefore the HDF (HL7 Development Framework) technique generates XML 
schema representation format based on HL7's Reference Model (RIM) and CDA structured document format.

HL7-CDA bear the hierarchical structure of XML-based medical information between the means, the part that defines the structure of the document, the written part is a separate and excellent scalability and flexibility.

\section{SYSTEM DESIGN}

System with accurate and easy-care patient response, information security, stability, high capability will be built into the system, you can share the work between hospitals can reduce the burden of the cost of Complex and diverse forms of health information data by systematic accumulation can provide the same level of medical services to all patients, to provide guidelines for a variety of condition-specific care and nursing work.

Data exchange based on CDA is covered with management of the hospital and medical facilities. In this paper, HL7-CDA based medical information more in the way of the existing data exchange and management is proposed to use clinical document interchange system. HL7-CDA uses XML sharing medical information or the format and content of clinical information and provides a flexible interface to binary objects, including the text as well as images with a variety of multimedia information. HL7 message segments or elements can be exchanged encoded in MIME format as shown IN Figure 3.

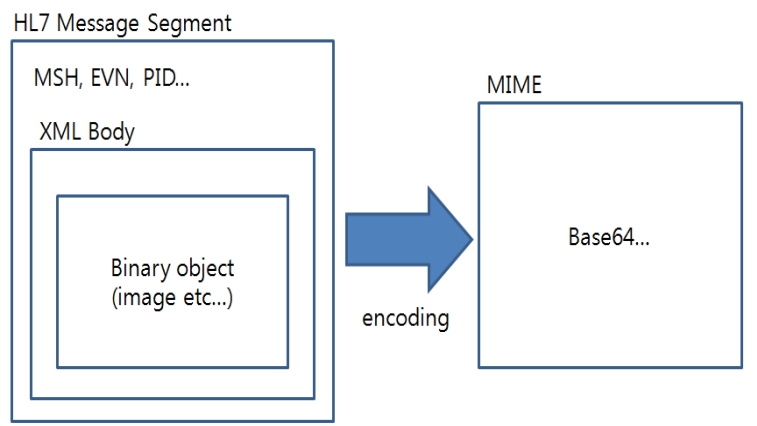

Figure 3. CDA document structure using MIME format

CDA documents is generated and stored in the form of a XML to transfer medical information and clinical information designed to database and system. CDA documents can be updated and deleted to store such as medical information and clinical information in the database and simultaneously sent to the external agency. CDA viewer outside agencies can see medical information and clinical information through the system.. Also the outside agency can transfer the data to other institutions in the same way as shown in Figure 4.

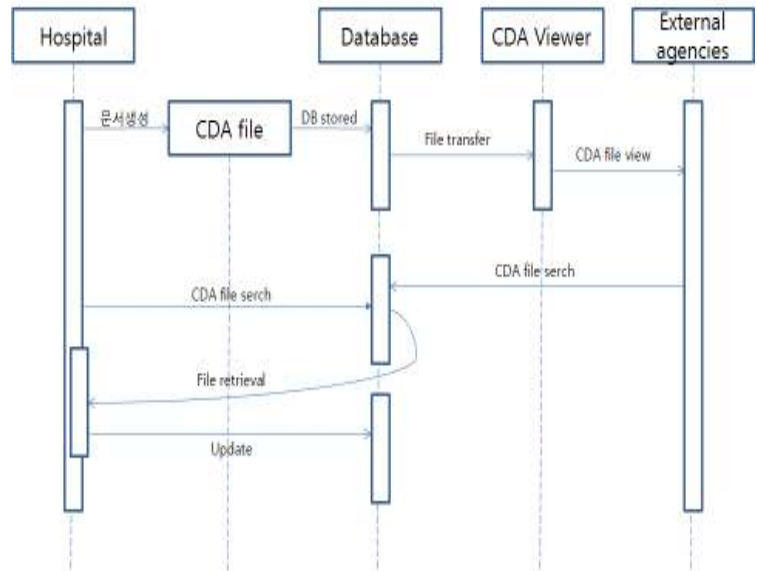

Figure 4. Sequence diagram for CDA document

\section{SYSTEM IMPLEMENTATION}

One document is stored in the form of XML, to build a schematic system for exchange CDA documents to care patients using medical history and information with writing program at each hospital. Structure of each agency is shown in Figure 5 for the CDA document interchange.

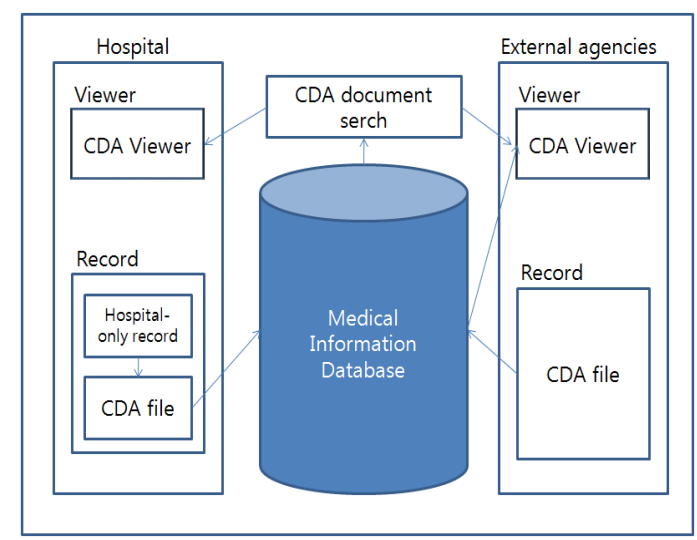

Figure 5. Structure for CDA document exchange system

Thereafter CDA documents are converted into an external agency or transfer to another hospital. Medical information records are converting the documents in the database. If necessary, documents are re-written at any time to be designed and viewed or deleted even if CDA document is available through the CDA Viewer. Medical documents exchanged during the document will be stored in the database of the system. Documents are not need to request or send because of less hassle, but not yet prepared a response packet loss that occurs during the exchange of documents. On the other hand, as security is important issue in clinical information, security and encryption techniques should be supplemented in the system. 


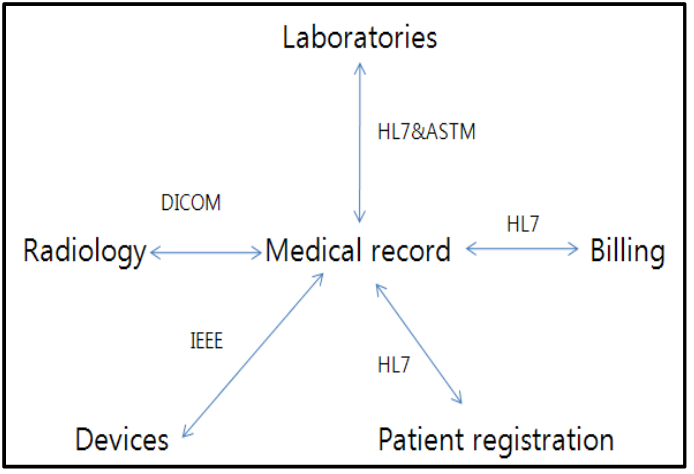

Figure 6. Health Information Standards interrelationships

Easy duplicate checks of hospitals belonging to the same doctor as well as the previously proposed hospital electronic chart system is built, the other doctors of the hospital computer you anytime, anywhere to identify the patient's condition and medical records, medical institutions between the exchange can be avoided. This makes it possible to remote medical system and medical institutions between the Interdisciplinary Being able to take advantage of the infinity of patients with a wide range of information than what is the biggest attraction of this system. Data easier to take advantage of the $\mathrm{Bu}$ Medical Research and patient characteristics categorized by medical institutions can take advantage of the management data. Research was focused on administrative tasks or completeness of the charts continuously increased to a large amount of medical data in a variety of medical records or to obtain useful information needed to improve the hospital management can focus on. In addition, to reduce the burden of the cost of the patient, the patient's benefit can be achieved. Chart of managed dedicated workers can be fed back to a more productive work, so work efficiency is increased significantly. The chart carriage dedicated personnel to strengthen the hospital's operating or service improvement work can be fed, so you can contribute to increase the productivity of the work of the hospital.

\section{Conclusions}

Most important compatibility is required in stadards of electronic documents for exchange medical records between hospitals. In addition, the standard codes for the conversation to be recorded in the patients with various record is required to some extent. Because health insurance, prescription drugs, inspection of material efforts are needed to standardize, the first compatibility measures are database structuresand file formats. In this paper, HL7-CDA based on the medical institution is proposed to exchange text or images by using XML. For the mutual exchange of clinical documents using the HL7 CDA, it is need to build a multimedia information such as image-based medical information and clinical documents. To do this, database structure is need to be simple and clear, and stored data such as text of Word or Hangul or standard DBF file format, excel, quick book to provide all field for conversion. Domestic and international standards are need to be established to support it. Organizations can raise the standards of the ISO, HL7, ASTM, DICOM standardized data sharing data and transmission between domestic hospital or outside the hospitals. It is required to transfer of data standards to configure active support from the government. To exchange medical information with existing hospitals, more detailed information can be exchanged so that more convenient use medical information. The system can be designed and built for exchanging or sharing medical information. Mutual exchange of medical information in the near future must be complemented and continued. It could be important medical information exchange, but especially security issues are a lot more efficient cryptographic techniques. It requires for the secure exchange medical information.

\section{References}

[1] R. H. Dolin, L..Alschuler, C. Beebe, "The HL7 clinical document architecture," Proc AMIA Symp, pp.552-569, 2001.

[2] J. M. Ferranti, R. C. Musser, K. Kawamoto, "The Clinical Document Architecture and the Continuity of Care Record: A Critical Analysis," Journal of the American Medical Informatics Association, vol.13, no.3, pp.245-252, May / Jun 2006

[3] N. H. Kim, "Rotor fault detection system for inverter driven induction motor using current signals and an Encoder," Journal of the Institute of Webcasting, Internet and Telecommunication(IWIT), Vol. 10, No. 5, pp. 128-135, Oct. 2010.

[4] S. K. Dwivedi, "Power Quality Improvements and Sensor Reductions in Permanent Magnet Synchronous Drives," PhD. Thesis, IIT Delhi, 2006.

[5] Yong-Gyu Jung, Seung-Ho Lee and Ho Joong Sung, Effective Diagnostic Method Of Breast Cancer Data Using Decision Tree, Journal of IWIT (2010), Vol.10 No. 5 pp.57-62

[6] I.C Kim, Y.G Jung, Using Baysian Network to analyze Medical Data, LNAI2734, Springer-Verlag (2003), pp.317-327

[7] Yong Gyu Jung, Ki Young Lee and Myung Jae Lim, Discharge Decision for Post-Operative Patients, Proceedings of ICHIT (2010), pp.195-199

[8] Shmueli, G., Patel, N. R., and Bruce, P., Data Mining for Business Intelligence (2009)

[9] lan H. Witten and Eibe Frank, Data Mining, Addison Wesley (2005), pp.315-333 


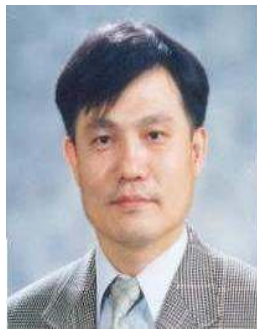

Yong Gyu Jung received the B.S. in physics Education from Seoul National University in 1981. And then he got the M.S. and ph.D. degree of Computer Science from Yonsei and Kyonggi University in 1994 and 2003 respectively. Since 1999, he has joined as a Faculty of Eulji University in dept. of Medical IT marketing. His research interests are in the areas of medical information analysis and international standards including e-Business. He is a Member of the UN/ECE/CEFACT and ISO/TC154 standard organization.

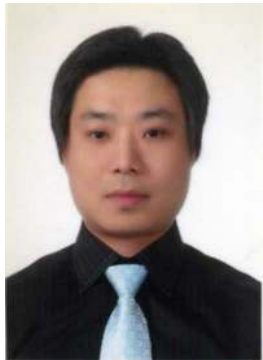

Young Ho Lee received the B.S. and M.S. at Suwon University in 2001 and 2003 respectively. And now he has joined as a researcher of COCif in Korea. His research interests are in the areas of $\mathrm{HCI}$ and SE. 